Proceedings of the 42th "Jaszowiec" International School and Conference on the Physics of Semiconductors, Wisła 2013

\title{
Superconductivity Study of GaN Highly Doped by Transition Metals
}

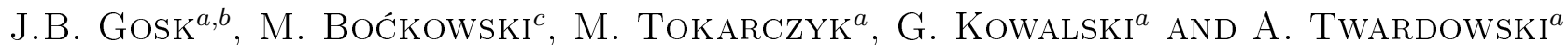 \\ ${ }^{a}$ Institute of Experimental Physics, Faculty of Physics, University of Warsaw, Hoża 69, 00-681 Warsaw, Poland \\ ${ }^{b}$ Faculty of Physics, Warsaw University of Technology, Koszykowa 75, 00-662 Warsaw, Poland \\ ${ }^{c}$ High Pressure Research Centre UNIPRESS, PAS, Sokołowska 29/37, 01-142 Warsaw, Poland
}

\begin{abstract}
$\mathrm{GaN}: \mathrm{Cr}$ and GaN:Fe single crystals as well as GaN:Mn micropowders highly doped by transition metals were grown to investigate low temperature superconductivity. Magnetic measurements revealed type I superconductivity with $T_{\mathrm{C}} \approx 6 \mathrm{~K}$ and $H_{\mathrm{C}} \approx 600 \mathrm{Oe}$, identical for all compounds and also identical to that observed before in $\mathrm{GaP}$ :Cr and GaAs:Cr. The presence of amorphous inclusions of gallium may explain existing superconductivity as a result of a phase transition leading to $\beta$-Ga during cooling down of the sample. Since the observed parameters are close to those characteristic for superconducting $\mathrm{Ga}(\mathrm{II})$ this possibility could not be ruled out.
\end{abstract}

DOI: 10.12693 /APhysPolA.124.877

PACS: 74.25.Ha, 74.70.-b, 74.25.Dw, 75.50.-y, 61.72.Dd, 81.10.Dn

\section{Introduction}

Gallium nitride, wide band energy gap semiconductor has been intensively investigated in last decade both by experimentalists and theoreticians. Despite great efforts the massive expectation were fulfilled only partly i.e., by its application in a blue laser system. But, the diluted magnetic semiconductors based on GaN still show the Curie temperature too low to be considered as an awaited spintronic material. There are still many questions about physical properties of $\mathrm{GaN}$ to be answered. The superconductivity of GaN was reported by different groups in time span of 50 years [1], but its origin is under vivid debate. At high pressure some semiconductors from the III-V group viz. GaAs, GaSb, and GaP show superconductivity [2]. On the contrary, there are no such experimental evidences in the case of $\mathrm{GaN}$, the compound from the same group. However, the transition to superconducting state at critical temperature $T_{\mathrm{C}} \approx 5.85 \mathrm{~K}$ at atmospheric pressure in $\mathrm{GaN}$ powders was reported by Alekseevskii and coworkers [1]. The results of this work were questioned by Rabanau and Berben [3] who did not observe any evidences of superconductivity above $1.5 \mathrm{~K}$ in GaN obtained by various preparation methods.

To clarify the controversy Kravchenko with coworkers synthesized two kinds of GaN powders: (i) at high temperatures (1350-1400 K) similar as in [1] and (ii) at low temperatures $(650-700 \mathrm{~K})$ with the use of mineralizer and performed investigation in search for superconductivity [4]. Authors managed to observe superconductivity with $T_{\mathrm{C}}=6.2 \mathrm{~K}$ but only in those samples synthesized in low temperatures. It was attributed to $\approx 1 \mu \mathrm{m} \beta$-Ga droplets (about $1 \%$ in volume). Some evidences of presence of $\gamma-\mathrm{Ga}\left(T_{\mathrm{C}}=7.3 \mathrm{~K}\right)$ and $\delta$-Ga $\left(T_{\mathrm{C}}=7.7 \mathrm{~K}\right)$ in the samples were also shown. Recently, the weak type-II superconductivity in GaN nanoceramics synthesized from nanocrystalline powders sintered at $1050^{\circ} \mathrm{C}$ with $T_{\mathrm{C}}=6.5 \mathrm{~K}$ has been reported [5]. In this work the superconductivity behavior was revealed in the strongly deformed GaN nanopowder (6.5 nm size grains) and tentatively explained by gallium precipitation most probably located between grains surfaces. Lately, superconductivity in Ga-based III-V semiconductors doped with transition metals was observed. Interestingly, bulk single crystals $\mathrm{GaP}$ and GaAs highly doped with $\mathrm{Cr}$ grown by liquid encapsulated Czochralski (LEC) method, revealed type I superconductivity with $T_{\mathrm{c}} \approx 6.2 \mathrm{~K}$ and $H_{\mathrm{c}} \approx 600 \mathrm{Oe}[6]$, thus very similar to that observed in $\mathrm{GaN}: \mathrm{Mn}, \mathrm{GaN}: \mathrm{Cr}$, and $\mathrm{GaN}: \mathrm{Fe}$, which are the subject of this paper.

\section{Samples}

Here we report on observation of superconductivity in GaN:TM (transition metal) obtained by different fabrication techniques which were in the form of single crystals as well as micro-powders. GaN:Mn micro-powders were obtained by ammonothermal method at the University of Warsaw [7]. The growth of investigated samples (GaMnN \#I [8]) was carried out in high pressure autoclaves at $p=5 \mathrm{kbar}$ and $T=500^{\circ} \mathrm{C}$ with the $\mathrm{KNH}_{2}$ mineralizer. The use of $\mathrm{KNH}_{2}$ mineralizer contrary to $\mathrm{LiNH}_{2}$ and $\mathrm{NaNH}_{2}$ ones results in samples with highest Mn concentration amounting to $1 \%$ but also with high structural disorder. The X-ray diffraction patterns revealed broadened reflections from the hexagonal structure with considerable diffused background complemented by small but distinct additional sharp peaks. Generally structure was considered as hexagonal one with high density of stacking faults (presumably cubic layers and/or lamellas of cubic layers) [8].

The GaN:Cr and GaN:Fe single crystals were synthesized from solution of nitrogen of liquid gallium under high pressure $p \approx 1.5 \mathrm{GPa}$ of $\mathrm{N}_{2}$ and elevated temperatures $T \approx 1500{ }^{\circ} \mathrm{C}$ at UNIPRESS [9]. GaN:Cr and $\mathrm{GaN}: \mathrm{Fe}$ were in the form of plates with diameter of the order of few millimeters. It should be stressed here that 
this growth method is known to produce gallium and graphite inclusions. The GaN:Cr and GaN:Fe crystals have wurtzite structure with $c$-axis perpendicular to the samples crystal plane. Due to higher solubility the concentration of $\mathrm{Cr}$ in $\mathrm{GaN}$ :Cr was much higher than the one of Fe in GaN:Fe. Also mean volume size of GaN:Cr plates was larger than that of GaN:Fe. Except for the low temperature range $(T<7 \mathrm{~K})$, all the investigated samples showed in addition to the expected two dominant phases i.e., diamagnetic (GaN lattice) and paramagnetic (PM) also ferromagnetic one (FM). Presence of FM is typical for this group of crystals and results from precipitates/ clusters when too high amount of TM is intended to be incorporated into GaN during the growth process.

\section{Results}

\subsection{Magnetic measurements}

Magnetic properties i.e., dc magnetization and ac susceptibility of all samples were performed by means of SQUID Quantum Design MPMS XL magnetometer. The dc magnetization of all samples was measured as a function of magnetic field (up to $7 \mathrm{~T}$ ) and temperature (1.9$400 \mathrm{~K}$ ). For all measured samples, magnetization corrected for diamagnetic constant of GaN lattice has revealed the dominant typical Brillouin paramagnetic contribution. Since, FM contribution was present in both $\mathrm{GaN}: \mathrm{Cr}$ and Ga:Fe crystals, therefore for search of superconductivity the samples with the lowest FM contribution were selected. The extended measurements of magnetization as a function of magnetic field at fixed temperatures in the range between $1.9 \mathrm{~K}$ and $12 \mathrm{~K}$ were performed. The collected data for $\mathrm{GaN}$ :Cr shown in Fig. 1 exemplify obtained results. The data are corrected for resultant contributions: the diamagnetic (GaN lattice), $\mathrm{PM}$ and FM to the total measured magnetization. For each temperature (see Fig. 1) characteristic diamagnetic response to external magnetic field is observed; magnetization decreases linearly with increasing magnetic field to its minimum and then relatively sharp increase of diamagnetic response follows. The minimum is decreasing with increasing temperature and its position is shifted towards lower magnetic fields. This characteristic behavior of diamagnetic response and decrease of relevant $T_{\mathrm{C}}$ are fingerprints of superconductive phase of any material.

Figure 2 presents ac susceptibility data for $\mathrm{GaN}: \mathrm{Cr}$ sample for which very weak dc signal from superconducting phase was observed. The real part of susceptibility collected in the temperature range between 2 and $12 \mathrm{~K}$ reveals a sharp transition to the superconducting state clearly visible at temperature about $6 \mathrm{~K}$. However, the volume fraction of superconducting state is at least three orders of magnitude smaller than the one of ideal superconductor and differs quite a lot among investigated samples.

The $H_{\mathrm{C}}$ dependence on temperature for the three investigated compounds i.e., GaN:Cr, GaN:Fe, and GaN:Mn is shown in Fig. 3.

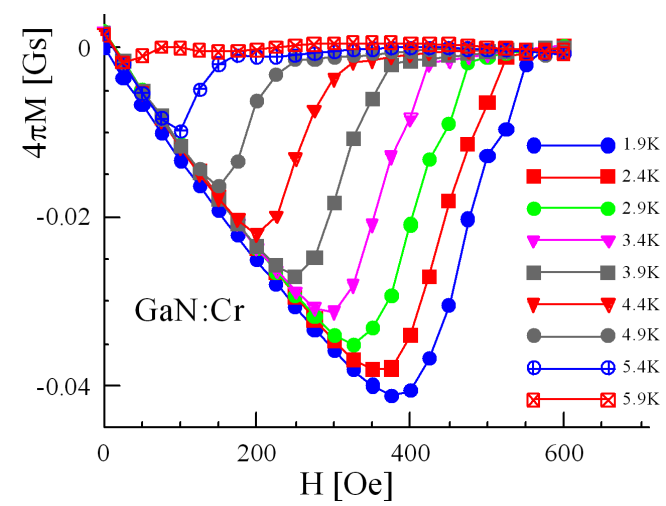

Fig. 1. Field dependence of magnetization of $\mathrm{GaN}: \mathrm{Cr}$ at fixed temperatures measured with increasing magnetic field. Small shift for curves measured for $T>$ $1.9 \mathrm{~K}$ comes from presence of non-vanishing $M_{\mathrm{r}}$ of FM contribution (first measurement - curve at $1.9 \mathrm{~K}$ and $\left.B_{\max }=800 \mathrm{Oe}\right)$.

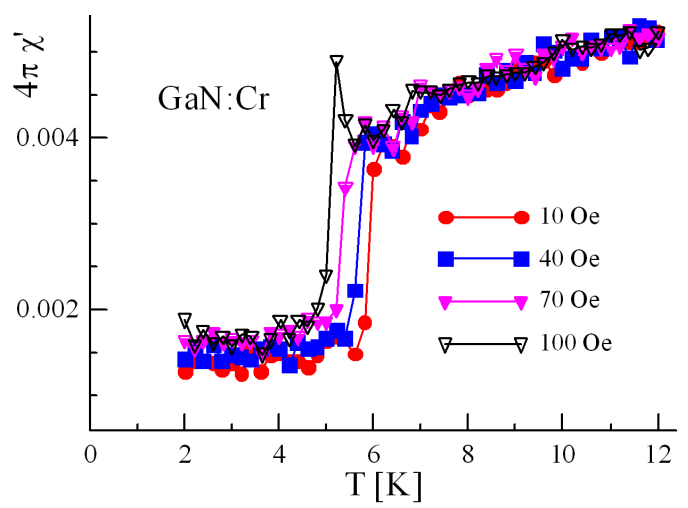

Fig. 2. Real part of the ac susceptibility of $\mathrm{GaN}$ : $\mathrm{Cr}$ measured with increasing temperatures at a frequency $1 \mathrm{kHz}$ and field amplitude 3 Oe for a fixed dc field subsequently equal to: $10,40,70$ and 100 Oe. No correction for PM, FM and diamagnetic (GaN lattice) contributions was made.

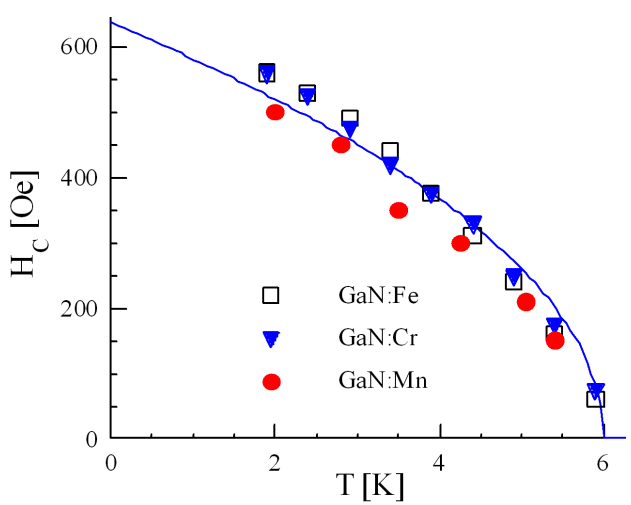

Fig. 3. The critical field dependence on temperature for GaN:Cr, GaN:Fe single crystals and GaN:Mn micro-powders. 


\subsection{X-ray measurements}

Since the origin of the superconducting state observed in samples is connected with presence of precipitates of gallium and relevant structural disorder (caused by excess of TM dopants incorporated in growth process [7]) the supplementary structural X-ray investigation were performed. GaN:Cr and GaN:Fe bulk samples were examined with Multi Crystal High Resolution X-ray Diffractometer. Diffractometer was equipped with the Hart-Bartels 4-bounce beam conditioner and also 2 bounce diffracted beam analyzer. Both "rocking curves" and reciprocal space maps were registered for 0002 relp (reciprocal lattice point). Lattice parameter $c$ (along 0001 direction) of the samples representing hexagonal system was also measured with the accuracy $10^{-4}[10]$ as compared with the reference sample of Si crystal measured by the Bond method [11].

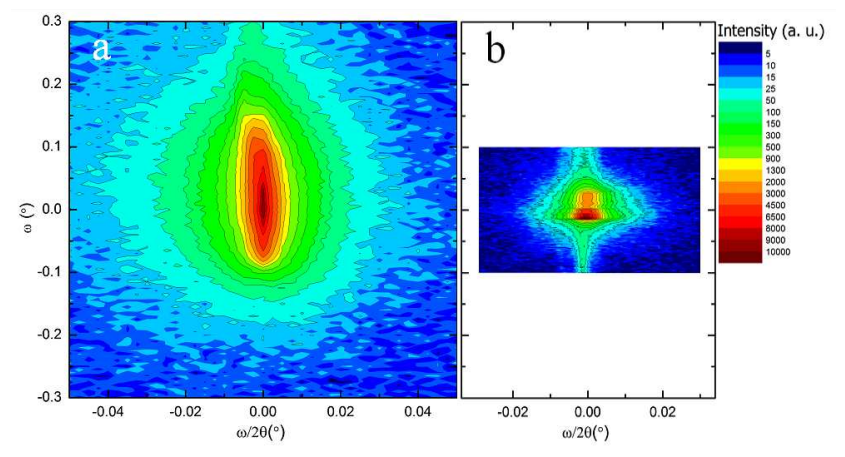

Fig. 4. Reciprocal space map (angular coordinates). (a) The GaN:Fe bulk crystal. 002 relp. Crystal lattice shows significant mosaic tilt as well as overall large contribution from diffuse scattering possibly from point like defects. (b) The GaN:Cr bulk crystal. 002 relp. Crystal lattice shows very little of mosaic tilt. Diffuse scattering surrounding the central feature is significantly smaller than for GaN:Fe. Note that angular scales of (a) and (b) are exactly the same to allow for the direct comparison of the both maps.

Recorded reciprocal space maps (Fig. 4a,b) and rocking curves clearly show that both crystals represent different states of the crystal lattice. GaN:Fe is showing high mosaic spread as well as high diffuse scattering. That is probably due to large concentration of the point-like defects. Mosaic tilt of the (0001) basal hexagonal lattice planes is spreading about 0.2 of the deg for Fe doped sample. Crystal lattice of the investigated GaN:Cr sample consists basically, of the two subgrains with dominating volume of one of them which is clearly seen as the single dominating peak in reciprocal space map (Fig. 4b). Both subcrystals are diffracting almost independently with the shift in the maximum position of about $0.025 \mathrm{deg}$. That could be due to the growth incident resulting in two subcrystal lattices. For both crystals $\mathrm{GaN}: \mathrm{Cr}$ and $\mathrm{GaN}: \mathrm{Fe}$ horizontal spread of the recorded intensities of main maxima on the reciprocal space maps (i.e., the $c$ axis lattice

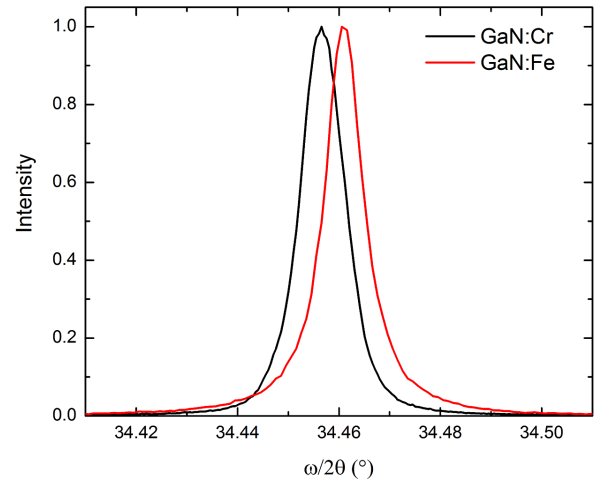

Fig. 5. Diffractometric $\theta-2 \theta$ scan of the both bulk crystals. It is clearly seen that there is a $c$ lattice parameter difference between the both samples.

parameter gradient) is almost the same. The $c$ lattice parameter values were established by diffractometric $\theta-2 \theta$ scans.

Recorded intensities (Fig. 5) show similar FWHM's for both crystals but different angular positions of the maximum. This clearly shows that $c$-lattice parameter is significantly different for both crystals. Since the angular scale was calibrated by the reference sample we can put absolute values for the both $c$-axis lattice parameters. It gives for the GaN:Cr $c=5.2015 \AA$. For the GaN:Fe $c=5.2008 \AA$. Fe doped sample has got the value closer to the undoped $\mathrm{GaN}$ value $(c=5.1851 \AA)$. If we disregard the bit of the twinning for the GaN:Cr crystal sample one can conclude that $\mathrm{Cr}$ doped sample presents overall good quality single crystal with low content of point like defects and no significant lattice tilts.

\section{Discussion}

For comparison the phase diagrams for GaN doped with TM as well as two other compounds from III-V group viz. GaP:Cr and GaAs:Cr measured before [6] are shown in Fig. 6. It is readily seen from the figure that the superconducting phase diagrams determined for $\mathrm{GaP}: \mathrm{Cr}$ and GaAs:Cr [6] are very similar to those of $\mathrm{GaN}: \mathrm{Cr}$, $\mathrm{GaN}: \mathrm{Fe}$, and GaN:Mn. Obviously, the difference in physical properties for these compounds is sufficient to expect observable difference in their phase diagrams. Lack of such differences suggests a common origin of the observed superconductivity in all investigated compounds. In our opinion, in accord with the work of Kravchenko [4], the source of the observed superconductivity is related to gallium. The TM dopant in Ga based III-V compounds plays a crucial role as a "catalyst" for the Ga precipitation process, analogous to high pressure treatment described in [5]. Thus our present observation concerning GaN:Fe, GaN:Cr, and GaN:Mn in connection to those concerning $\mathrm{GaAs}: \mathrm{Cr}$ and $\mathrm{GaP}: \mathrm{Cr}$ strongly suggests that the superconductivity in all these samples could be explained by the presence of amorphous inclusions of gallium and the 


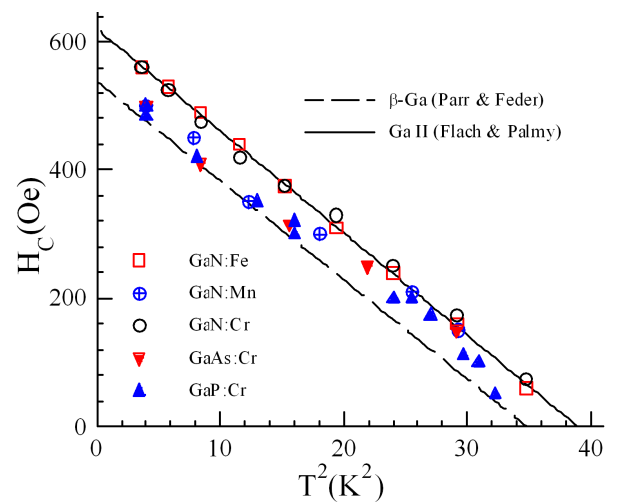

Fig. 6. Phase diagram for GaN:Cr, GaN:Fe, GaN:Mn, GaP:Cr, and GaAs:Cr [6] presented as $H$ versus $T^{2}$ dependence. The dash line corresponds to the data obtained by Parr and Feder for $\beta$-Ga phase [14] and solid line to the data obtained by Flach and Palmy for high-pressure superconducting phase $\mathrm{Ga}(\mathrm{II})$ [15].

phase transition leading to a formation of $\beta$-Ga during cooling down the sample [6]. It is worth to recall here that almost all of the polymorphic modifications of gallium are reported to exhibit superconductivity, and Ga in amorphous state is also superconducting with $T_{\mathrm{c}}$ equal to 8.5 K. The so-called $\alpha-\mathrm{Ga}, \gamma-\mathrm{Ga}$ and $\delta$-Ga phases have $T_{\mathrm{c}}$ equal to: $1.1 \mathrm{~K}, 7 \mathrm{~K}$ and $7.85 \mathrm{~K}$, respectively [12]. The transition temperature of $\beta$-Ga phase is reported to vary in the range 5.6-6.3 K [13]. For this phase Parr and Feder reported value of $H_{\mathrm{c}}=(538 \pm 5)$ Oe [14], quite close to that one observed in the current studies. However, since the observed parameters especially for $\mathrm{GaN}$ : $\mathrm{Cr}$ and GaN:Fe are very close to those characteristic for superconducting Ga II (see Fig. 6) this possibility should also be taken into account $[6,15]$.

\section{References}

[1] N.E. Alekseevskii, G.V. Samsonov, O.I. Shulishova, Zh. Eksp. Teor. Fiz. 44, 1413 (1963) [Sov. Phys. JETP-USSR 17, 950 (1963)].

[2] I.V. Berman, N.B. Brandt, V.I. Sidorov, Pisma Zh. Eksp. Teor. Fiz. 14, 18 (1971) [ Sov. Phys.-JETP Lett. 14, 11 (1971)]; L.F. Vereshchagin, E.N. Yakovlev, Yu.A. Timofeev, B.V. Vinogradov, Pisma Zh. Eksp. Teor. Fiz. 26, 61 (1977) [Sov. Phys.-JETP Lett. 26, 55 (1977)].

[3] A. Rabenau, T.J. Berben, Phys. Lett. 12, 167 (1964).

[4] V.S. Kravchenko, M.Z. Graifer, M.A. Starikov, A.G. Klimenko, Izv. Akad. Nauk SSSR, Neorg. Mater. 18, 1308 (1982) [ Bull. Acad. Sci. USSR, Inorg. Mater. 18, 1105 (1982)].

[5] A.J. Zaleski, M. Nyk, W. Strek, Appl. Phys. Lett 90, 042511 (2007).

[6] J.B. Gosk, R. Puzniak, G. Strzelecka, A. Materna, A. Hruban, A. Wisniewski, A. Szewczyk, G. Kowalski, K. Korona, M. Kaminska, A. Twardowski, Supercond. Sci. Technol. 21, 065019 (2008); J.B. Gosk, G. Strzelecka, G. Kowalski, A. Hruban, M. Kamińska, A. Twardowski, J. Gronkowski, Acta Phys. Pol. A 110 , 189 (2006)

[7] M. Zając, J. Gosk, E. Grzanka, S. Stelmakh, M. Palczewska, A. Wysmołek, K. Korona, M. Kamińska, A. Twardowski, J. Alloys Comp. 456, 324 (2008).

[8] M. Zając, Ph.D. Thesis, University of Warsaw, 2007.

[9] S. Krukowski, M. Bockowski. B. Lucznik, I. Grzegory, S. Porowski, T. Suski, Z. Romanowski, J. Phys., Condens Matter 13, 8881 (2001).

[10] D.K. Bowen, B.K. Tanner, in: X-ray Metrology in Semiconductor Manufacturing, CRC-Taylor \& Francis, Boca Raton 2006, p. 57.

[11] K. Wokulska, in: The precise measurement of lattice parameters and their application to structure investigation of solid solutions crystals, Wydawnictwo Uniwersytetu Śląskiego, Katowice 1997, p. 43 (in Polish).

[12] N.E. Phillips, Phys. Rev. 134, A385 (1964).

[13] D.R. Lide, Handbook of Chemistry and Physics, 71th ed., Chemical Rubber, Cleveland 1991.

[14] H. Parr, J. Feder, Phys. Rev. B 7, 166 (1973).

[15] R. Flach, C. Palmy, Phys. Lett. A 29, 545 (1969). 